\title{
SELECTED SUMMARY OF DISCUSSIONS
}

\author{
David L. Spooner and Martin S. Olivier
}

\section{RECENT ADVANCES IN INFORMATION SYSTEMS SECURITY, JAJODIA}

Rosenthal: Can you partition the world into objects requiring different types of access control policies?

Jajodia: The real world needs different types of policies. Currently this is often done within application systems. We would like to be able to do it in the operating system.

Rosenthal: Administration of access control policies may be the biggest impediment in the real world.

Rosenthal: Can you have different policies on the same objects?

Jajodia: You could do it, but it would be very complex.

Samarati: Using logic rules to represent policies, you can do it.

Rosenthal: Few database administrators would be able to handle the specification of such access control policies. An important research question is how can we make specification of access control policies easier. 
Rosenthal: Are security rules with imperatives (e.g., add a proprietary notice to a returned document) a good idea? This could cause things to happen with the user unaware and which the user could consider undesirable.

Jajodia: The owner of an object must understand the policy being enforced for that object. This idea still needs some work.

\section{ROLE-BASED ACCESS CONTROL ON THE WEB USING LDAP, PARK, AHN, AND SANDHU}

Tan: Suppose the link between the Role Server and Web Server breaks. In the Server-Pull case, the user would not be able to access anything. What about in the UserPull case?

Park: If the user gets his/her roles from the Role Server before the link breaks, then the user can still access the web. Therefore, the User-Pull case is less vulnerable to a single point of failure.

Audience: One can have redundant Role Servers to minimize this problem.

Ting: You must be careful in the User-Pull case that the user cannot modify the roles that he/she gets from the Role Server.

Gudes: You have a role hierarchy. How much of the hierarchy must be pulled to the user or the Web Server?

Sandhu: You must pull the entire relevant path in the hierarchy.

Rosenthal: What does the end-user need to know to use your system?

Park: In the User-Pull case, the user needs to know about the Role Server.

Rosenthal: Most users won't want to have to know about the Role Server. 


\section{CONSTRAINTS-BASED ACCESS CONTROL,} TAN

Rosenthal: Doesn't the Turing completeness of the constraints make them hard to verify?

Tan: $\quad$ Yes, but subsets are verifiable.

Samarati: The predicates that you use in your rules don't appear to be predefined in your language. Therefore, how does this differ from previous work using logic rules?

Tan: We are working now to define a small set of predicates for the language.

Samarati: Do you keep a list of things that have been revoked?

Tan: Yes.

Rosenthal: There is a general problem of using a database of access control rules to control access to a database. Using logic rules is a good way to frame this problem. Is it better to use logic to implement the access controls or is it better to use the database system itself? The answer in general is not clear.

Gudes: This approach is useful for verifying the validity of an access request.

Rosenthal: You might use this approach to explore what happens when access polices are embedded in logic. Which is better, an efficient SQL-based system or a less efficient logic based system? Maybe security will be the killer application for logic databases.

Tan: We must figure out how to constrain the language so that it always works efficiently.

Jajodia: This is all fine as long as logic doesn't make the specification of policies harder.

Sandhu: This is yet another language that programmers will need to learn. 
4. SUBJECT SWITCHING ALGORITHMS FOR ACCESS CONTROL IN FEDERATED DATABASES, YANG, WIJESEKERA, AND JAJODIA

Rosenthal: Do you see this as an interactive tool for use during schema design, a decision aid?

Wijesekera: Yes.

Rosenthal: There are some similarities here to work done on role comparisons and merging by Sylvia Osborn and her students.

5. EFFICIENT DAMAGE ASSESSMENT AND REPAIR IN RESILIENT DISTRIBUTED DATABASE SYSTEMS, LIU AND HAO

Jajodia: What happens when a site fails?

Liu: You cannot afford to wait for it to recover. One is forced to leave the damaged site damaged.

Jajodia: In this case, damage can spread.

Audience: How do you make sure that repairs to a database are faster than the transactions executing against the database?

Liu: One technique is to use priorities on transactions.

6. STATE-DEPENDENT SECURITY DECISIONS FOR DISTRIBUTED OBJECT-SYSTEMS, BISKUP AND LEINEWEBER

Audience: Can the final state from one decision be the same as the initial state for the next one? 
Leineweber: Yes, but it is better to separate them to keep the definitions cleaner.

\section{REORGANIZATION OF DATABASE LOG FOR INFORMATION WARFARE DATA RECOVERY, SOBHAN AND PANDA}

Panda: We need the source code logic to implement redo in the $\log$.

Rosenthal: It will be difficult to get people to use only languages that your system supports so that you can process the source code logic.

Liu: The logic is needed in this approach because it maintains the original order of the transactions.

Rosenthal: Source code logic may not be available for all transactions.

Jajodia: Maintaining the original serial order of the transaction requires much more work than other approaches.

Liu: $\quad$ Redo may kill some transactions since their preconditions are no longer met.

8. RANDOMLY ROVING AGENTS FOR INTRUSION DETECTION, MOSKOWITZ, KANG, CHANG, AND LONGDON

Jajodia: Can you give us an example of an attack that your agents will detect?

Kang: A checksum attach is an example.

9. PUBLIC TELEPHONE NETWORK (PTN) VULNERABILITIES, LORENZ, KELLER, MANES, HALE AND SHENOI 
Reid: Do you have the differences between optical fiber and copper in mind?

Lorenz: Those are hardware issues. The taxonomy deals with software threats.

\section{FLEXIBLE SECURITY POLICIES IN SQL,} BARKER AND ROSENTHAL

Gudes: How does the translation algorithm work?

Rosenthal: Stage 1: translate to SQL (without materialization). Stage 2: decide what to materialize. Step 2 is based on guesses. One therefore needs experimental results upon which to base a decision for step 2 .

11. THE INFERENCE PROBLEM AND UPDATES IN RELATIONAL DATABASES, FARKAS, TOLAND, AND EASTMAN

Rosenthal: A 2\% improvement may not give much protection.

Farkas: Results are application-dependent.

Rosenthal: Have people tried to do this sort of thing in real systems?

Jajodia: No, the closest is statistical databases.

Rosenthal: It is likely that few organizations will be willing to pay the overhead.

Farkas: The approach can be made more efficient and then some companies might be willing to use it.

Rosenthal: The cost-benefit analysis may be weak in most cases.

Jajodia: It could be used for the web where you consider that you have a single user (i.e., the general public).

Rosenthal: I agree that we need to do inference analysis.

Spalka: Does requesting all information in one query circumvent security? 
Farkas: No, related items will not be released together.

Audience: It appears that your approach cannot deal with an attack from multiple collaborating users.

Farkas: This is future work. One idea is to monitor groups of users as a single user.

Jajodia: We don't know how things will turn out in the future, so this sort of work could be important.

\section{MANAGING CLASSIFIED DOCUMENTS IN A RELATIONAL DATABASE, SPALKA}

Samarati: In your approach, when you lie, a high user must login as high to delete the high tuple, then logout, and finally login again at the low level to delete the lie. Polyinstantiation takes care of this automatically.

Spalka: A high user needs to remember lies and when to delete them. There is a trade-off between easy of use and level of confidentiality.

\section{ODAM: AN ON-THE-FLY DAMAGE ASSESSMENT AND REPAIR SYSTEM FOR COMMERCIAL DATABASE APPLICATIONS, LUENAM AND LIU}

Mukkamala: The approach doesn't appear to capture other types of dependencies such as semantic dependencies.

Jajodia: Semantic dependencies are difficult to capture.

Farkas: Cleaning transactions cause a significant performance penalty when there are lots of dependencies.

Liu: The use of a proxy is the biggest reason for this.

Farkas: Would it be better to stop the database and clean it periodically rather than continuously slowing it down? 
Liu: $\quad$ Many applications do not want the database to be halted.

Ting: What do you do about users who get the wrong answer?

Liu: We are concerned about this, but we can't do much about it. There are problems at both the logical and the physical levels. We deal here with only the physical level.

\section{PANEL: XML DATABASES - ARE ALL THE PROBLEMS SOLVED?}

Osborn: $\quad \mathrm{XML}$ is similar to the old hierarchical databases. Maybe we can leverage security concepts from old hierarchical database systems.

Rosenthal: As query languages improve for XML databases, we should be able to do more and more with the queries themselves.

Samarati: It is not clear how to make decisions when queries overlap. Negative authorizations can be a problem. It may be necessary to translate to finer grain objects to make decisions.

Thuraisingham: How similar are XML databases to object-oriented databases.

Samarati: There are similarities and differences.

Ting: $\quad \mathrm{XML}$ is replacing the Electronic Data Interchange (EDI) format for electronic commerce. Certification and authentication of documents is a real problem.

Thuraisingham: eBXML is designed to handle electronic commerce applications.

Rosenthal: In many cases, required access controls should be the same regardless of the document type.

Samarati: You may want to encrypt or sign different parts of a document separately. 
Gudes: XML isn't really a hierarchy but is more a graph. So you really need authorization based on path expressions.

Samarati: This would be true if you allow cross-links between documents that have different access control requirements. However, in practice you often don't allow cross-links in such cases.

Gudes: Does any research work consider these issues?

Samarati: The work of Samarati, Bertino and Jajodia ["An Authorization Model for a Distributed Hypertext System," IEEE Transactions on Knowledge and Data Engineering, August 1996, Vol. 8, No. 4, pp. 555-562.] considers this. You usually don't want propagation across links between documents.

Rosenthal: XML captures more of the real world complexity and we need to exploit this. It provides both elegance and simplicity. Using queries and views of XML documents may be one way to pull the concepts together into one framework.

Samarati: Views are not necessarily simpler and may be less powerful.

Rosenthal: These issues are worth exploration by the research community.

\section{A COMPARISON BETWEEN CONSA AND CURRENT LINUX SECURITY IMPLEMENTATIONS, HARDY AND OLIVIER}

Gudes: Do you view your work as a front-end to heterogeneous systems?

Hardy: That is one way to use it. It can also be used by applications to enforce application security.

Atluri: Do you assume that security levels are hierarchical in LOMAC?

Hardy: Yes. 
Rosenthal: It should also work with a lattice of security classifications.

Hardy: It was originally intended for operating systems where a hierarchy is usually sufficient.

Olivier: The original paper shows that it can handle a lattice.

16. A NOVEL APPROACH TO CERTIFICATION REVOCATION MANAGEMENT, MUKKAMALA AND JAJODIA

Rosenthal: Managing CA's is extra overhead.

Rosenthal: This is similar to the web caching problem. Trusted replication is an interesting problem and is a general instance of this problem.

Audience: What is the communications overhead?

Jajodia: There is a cost going to a CAD and you may not always need the most up to date data. In this case, if you are not willing to pay the extra cost each time, you can use the CA.

Gudes: What happens if a CA is revoked?

Mukkamala: Users must go back and get new certificates.

\section{AN EXTENDED TRANSACTION CONTROL MODEL APPROACH FOR MULTILEVEL SECURE TRANSACTION PROCESSING, ATLURI AND MUKKAMALA}

Farkas: Have you compared your work to that of Ray and Jajodia?

Atluri: They do not make provision for value constraints. 


\section{REGULATING ACCESS TO XML DOCUMENTS, GABILLON AND BRUNO}

Spalka: How do you know when you need a cover story?

Gabillon: It is not like cover stories in mandatory access control. You decide when you want to use a cover story; the system gives you no assistance.

Jajodia: There is a distinction between a provision and an obligation. A provision is an action that your security server takes. An obligation is part of the data received by the user.

Gudes: Can you compare your system to other systems?

Gabillon: The semantics are different. In some systems, nodes can't be independently protected. For example, text nodes can't be independently protected. We have no propagation policy. There are different conflict resolution policies. We use priorities. We use XSLT to build the system. Other prototypes use Java. As a result we had to do much less programming. 